\title{
The Interventional Radiology Service During the COVID-19 Pandemic: Steps for Managing the Risk of Infection
}

\author{
Anna Maria lerardi $\mathrm{MD}^{1}$, Aldo Carnevale $\mathrm{MD}^{2}$, Melchiore Giganti MD PhD \\ and Gianpaolo Carrafiello MD PhD ${ }^{1,4}$ \\ 'Radiology Department, Fondazione IRCCS Cà Granda, Ospedale Maggiore Policlinico, Milan, Italy \\ 2University Radiology Unit, Radiology Department, Arcispedale Sant'Anna, Ferrara, Italy \\ ${ }^{3}$ Department of Morphology, Surgery and Experimental Medicine, Radiology Section, University of Ferrara, Ferrara, Italy \\ ${ }^{4}$ Department of Health Sciences, Università degli Studi di Milano, Milan, Italy
}

\begin{abstract}
It is imperative to ensure the safety of health-care workers in the angiographic room during the outbreak of the coronavirus disease 2019 (COVID-19). The selection criteria for interventional radiology (IR) procedures, the preparation of the staff and angiographic suite, ventilation systems, and intra- and post-procedural workflow optimization methods are detailed. The specific measures needed to protect occupational safety and health may result in higher costs, longer procedural times, and greater technical problems. However, these precautions may help to minimize the spread of COVID-19 among IR practitioners.
\end{abstract}

Keywords: Coronavirus Disease 2019; COVID-19; Interventional Radiology; Angio Suite

\section{INTRODUCTION}

Worldwide, health-care systems are facing a critical situation from the coronavirus disease 2019 (COVID-19) pandemic. It is mandatory that interventional radiology (IR) services, as a major arm of radiology departments and modern health care, can be appropriately provided under these extraordinary circumstances. Indeed, essential activities should be identified and prioritized in an effort to maintain continuity of service delivery; strategic shifts should be planned to provide maximum benefit for the patients using increasingly limited resources. These essential activities in IR settings account for the whole spectrum of emergency procedures, for instance embolization for bleeding control or endovascular treatment of stroke. Other non-deferrable interventions, such as those dedicated to critical health conditions in

\section{Corresponding author:}

Anna Maria lerardi, Unità Operativa di Radiologia, Fondazione I.R.C.C.S. Cà Granda Ospedale Maggiore Policlinico, Via Francesco Sforza 35, 20122 Milan, Italy.

Email: amierardi@yahoo.it

(C) 2020 CC BY 4.0 - in cooperation with Depts. of Cardiothoracic/ Vascular Surgery, General Surgery and Anesthesia, Örebro University Hospital and Örebro University, Sweden oncology, should also not be discontinued. However, the outbreak of the novel COVID-19 pandemic may find some IR services unprepared. This paper may help IR staff to navigate through these new challenges.

COVID-19 is officially a pandemic, first recognized in December 2019 in Wuhan, China. The disease was rapidly shown to be caused by a novel coronavirus that is structurally related to the virus causing severe acute respiratory syndrome (SARS), subsequently named SARS-CoV-2. An efficient coronavirus human-to-human transmission is the cause of the rapid spread of this outbreak; to date, in Italy over 100,000 cases [1] have been reported and almost half of these are registered in our area (Lombardy) [1,2]. The virus is most likely to be transmitted via respiratory secretions in the form of droplets or aerosols [3]; fomite transmission may constitute another method of contagion [3] since the virus may remain viable for hours to days depending on the type of contaminated surface [4].

Due to the high percentage of aerosol-transmitted infections and possible permanence of the virus on surfaces, the main public health preventive advice focuses on social distancing and hygiene. These general principles are also applied to health systems and, consequently, to IR units, in light of the risk of nosocomial spread of infection. Therefore, proper preparation is crucial to reduce 
exposure of health-care workers and other patients in IR. As long as possible, in regions with a heavy burden of the COVID-19 pandemic, every patient and healthcare provider should be screened through real-time reverse transcription polymerase chain reaction tests for the detection of SARS-CoV-2. Patients with uncertain diagnosis should be managed as infected ones, and preventive quarantine should be applied to health-care workers also uncertainly diagnosed.

Dedicated paths to the angiographic suite should be planned for the isolated patients, including designated elevators and routes when possible, in an effort to minimize contact with other patients and staff. Indeed, few IR services are provided with distinct outpatient, inpatient, and isolation facilities; otherwise, developing a work plan based on temporal segregation to different groups of patients may be a solution. Moreover, other departments equipped with portable fluoroscopic units (i.e. operating theaters) may be temporarily made available to IR practitioners.

\section{SELECTION OF THE PROCEDURES}

A robust planning and deep reorganization of the IR workflow are fundamental, largely driven by the severity of COVID-19 dissemination in the area and local policy. First, it is pivotal to determine which procedures are non-urgent and can be delayed or rescheduled. Essential procedures for life-threatening conditions (i.e. intravascular embolization for bleeding control, thrombectomy for stroke, acute mesenteric ischemia treatment, endovascular repair for aortic rupture), urgent treatments in oncology patients (percutaneous biliary drainage, percutaneous ablation and chemoembolization in selected cases, bridging therapies for liver transplant, and a few others), and treatment of time-sensitive conditions and organ-sparing procedures (urgent diabetic foot angioplasty, percutaneous nephrostomy, prophylactic occlusion balloon placement for the prevention of postpartum haemorrhage in patients with abnormal placental implantation) must be regularly delivered. Other procedures may be discussed in a multidisciplinary team based on a case-by-case perspective. When necessary, ultrasound (US)-guided interventions should be performed at the patient's bedside in their isolation room.

The use of bedside US in the patient's isolation room to guide procedures increases safety by reducing the nosocomial spread of COVID-19. Indeed, US should be considered the modality of choice to guide an increasing number of interventions. Before entering the isolation room, the US system must be covered using transparent plastic and the probe must be protected with a sterile probe cover. The covers must be removed after the end of the procedure, before leaving the room, and the machine must be cleaned with chlorhexidine-ethanol solutions.

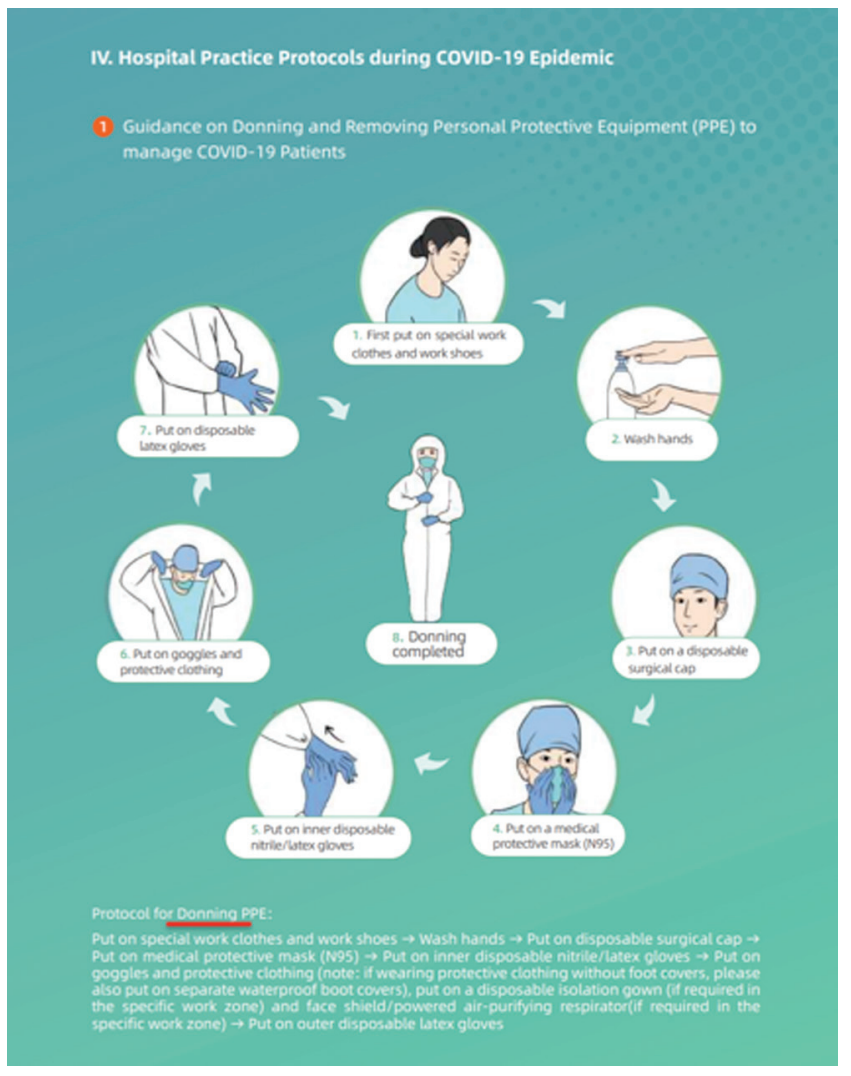

Figure 1 Guidance on donning personal protective equipment (PPE). From "Handbook of COVID-19 Prevention and Treatment". The First Affiliated Hospital, Zhejiang University School of Medicine. Compiled According to Clinical Experience. Tingbo Liang, Editor in Chief.

\section{PREPARATION}

Adequate personal protective equipment (PPE) is fundamental, and must be available on-hand and securely stored. Education on proper usage should be given to staff, particularly in the first phase of the emergence (Figure 1). All pre-procedural preparations should be completed before the arrival of the patient, aiming to minimize the unnecessary time spent in the department and possible contact with other patients and health-care professionals. Angiographic room staff should put on, use, take off, and dispose of PPE properly; this includes gowns, gloves, masks, eye protection, and shoe covers.

To avoid contamination, non-essential and mobile equipment needs to be removed from the angiographic room. Clean towels should be used to cover fixed and essential contact surfaces, whereas plastic should be preferred for control panels and changed between consecutive patients (Figure 2a,b). Proper cleaning supplies should be ensured for clean and contaminated work areas separately.

Other staff such as administrative workers should be kept at a safe distance from the patients in separate areas; they do not require PPE [5].

Health-care workers are in the front line of any outbreak response, and are subsequently exposed to hazards 

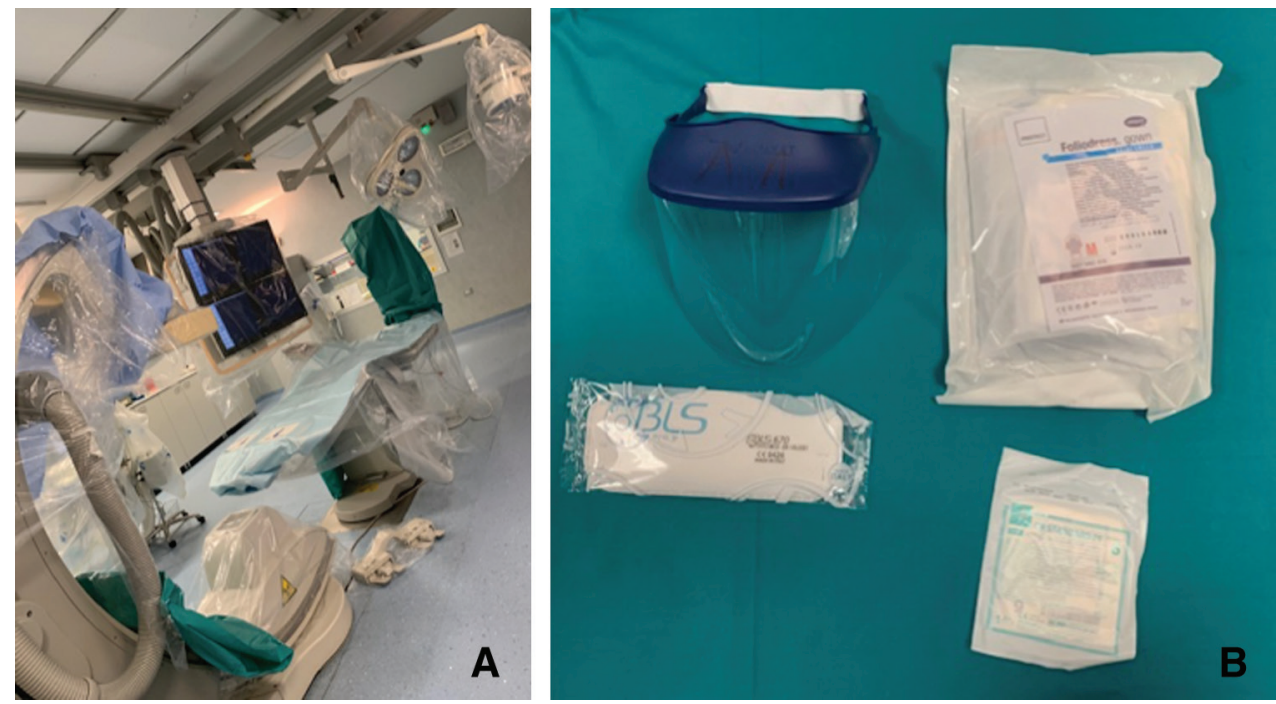

Figure 2 Angiosuite fully equipped before the arrival of the patient. (a) Fixed and essential contact surfaces covered with clear towels. (b) PPE of the operator, including eye protection and lead apron, face shield, sterile gown, and gloves.

that include not only exposure to infection, but also long working hours, psychological distress, and fatigue. Consequently, the work schedule for interventional radiologists should be replanned to protect occupational safety and health, and ensure likewise the delivery of essential services. Rotating shifts (possibly per week) may be implemented to limit their exposure and prevent infection.

\section{VENTILATION OF THE ANGIOGRAPHIC SUITE}

No dedicated ventilation systems are necessary in the angiographic suite, since SARS-CoV-2, based on the available evidence [3], presents human-to-human transmission through close contact and droplets, not by airborne transmission. Modern angiosuites are usually provided with ventilation systems similar to operating rooms; nevertheless, high-efficiency particulate air filtration systems are advisable when available.

\section{INTRA-PROCEDURAL MEASURES}

Adequate PPE is recommended by the World Health Organization (WHO) [5] (Figure 3). Notably, carrying out aerosol-generating procedures on COVID-19 patients requires N95 or FFP2 respirators or equivalent. In the angiographic suite these procedures include, for instance, percutaneous gastrostomy, naso-gastric or naso-jejunal feeding tube insertion, tracheal, esophageal, gastric, or duodenal dilatation or stenting, and bronchial artery embolization [6].

All non-intubated patients who are either infected, or suspected to be, must wear a FFP2 mask in the angiosuite.

The use of the N95 or FFP2 masks must be extended to operators standing at the patient's side during the

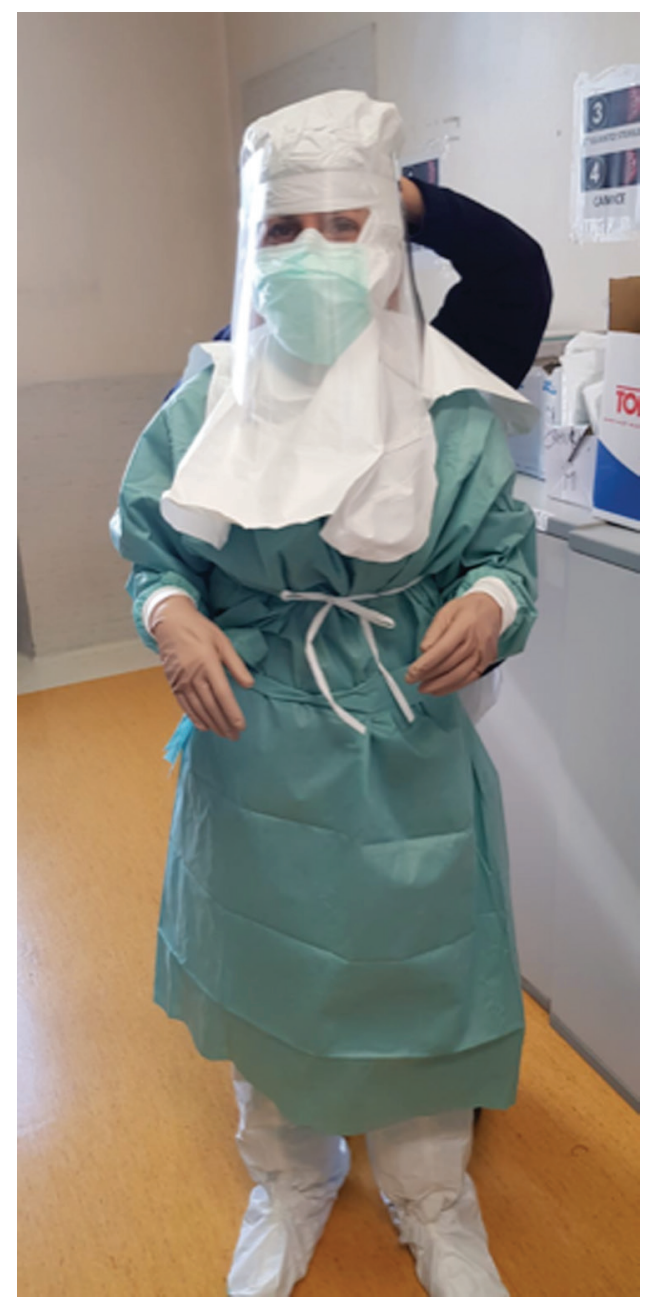

Figure 3 Operator during the preparation. 


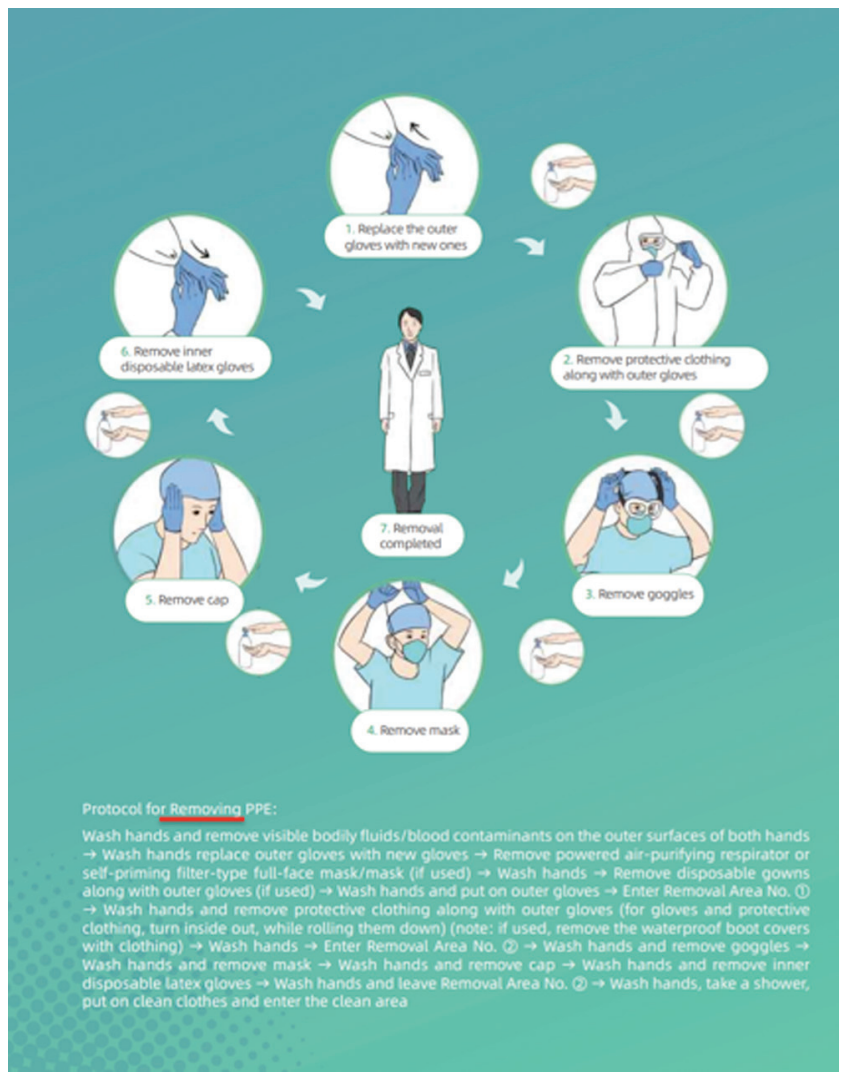

Figure 4 Guidance on removing PPE. From "Handbook of COVID-19 Prevention and Treatment". The First Affiliated Hospital, Zhejiang University School of Medicine. Compiled According to Clinical Experience. Tingbo Liang, Editor in Chief

procedure and giving specific instructions when the patient is non-intubated but is wearing an oxygen mask. During the entire procedure, the doors of the angiosuite must be kept closed. It may be advisable for the staff to stay inside the angiographic room, possibly behind lead screens, even during image acquisition. Indeed, it is essential to minimize in these circumstances the risk of cross-contamination of less adequately protected healthcare workers staying outside the intervention room. Therefore, to reduce movement in and out of the potentially contaminated room, the staff wearing appropriate PPE and sterile equipment must remain close to the angiographic bed, whereas a non-sterile radiographer with full PPE could help with the angiographic table control. Communication between operators in and out the room should be done by microphone.

The measures described are expected to increase the overall complexity and time of the procedure.

\section{POST-PROCEDURAL MEASURES}

Published literature suggests that SARS-CoV-2 is stable for days on certain surfaces under experimental conditions [3]. However, although fomite transmission is plausible, at present there is no robust evidence that supports infection through contaminated surfaces. These data may have profound implications for the implementation of rationale room decontamination procedures.

Appropriate education of staff and strict personal discipline are necessary.

It is strongly suggested that health-care workers remove PPE immediately after leaving the angiographic room, in order to avoid contamination of their colleagues or themselves; an adjacent small room may be used for the PPE removal process (Figure 4) and used PPE must be collected in dedicated disposal bags.

Access to reporting rooms is permitted only after PPE removal and proper hand hygiene.

Plans for terminal cleaning of procedure rooms must be ensured and cleaners themselves are required to wear proper PPE [5,6]. Exposed surfaces including imaging equipment in the angiosuite must be cleaned with $70 \%$ ethanol or chlorhexidine-ethanol solutions, and floors cleaned with approved disinfectants (i.e. sodium hypochlorite solutions). The whole cleanup procedure takes approximately $30 \mathrm{~min}$. Immediately after, the room needs to be ventilated for at least $30 \mathrm{~min}$, and then it is suggested that the room is left for a further $30 \mathrm{~min}$ with closed doors before the next intervention.

\section{CONCLUSION}

While health systems are strained worldwide by the outbreak of the COVID-19 pandemic, adequate control measures are fundamental to reduce to a minimum the nosocomial spread of this infection even in the IR services. The specific measures needed to protect occupational safety and health may result in higher costs, longer procedural times, and greater technical difficulties. However, these precautions can help to minimize the risk of infection by the novel coronavirus among IR practitioners.

\section{Ethics Statement}

(1) All the authors mentioned in the manuscript have agreed to authorship, read and approved the manuscript, and given consent for submission and subsequent publication of the manuscript.

(2) The authors declare that they have read and abided by the JEVTM statement of ethical standards including rules of informed consent and ethical committee approval as stated in the article.

\section{Conflicts of Interest}

The authors declare that they have no conflicts of interest.

\section{Funding}

The authors received no financial support for the research, authorship, and/or publication of this article. 


\section{REFERENCES}

[1] World Health Organisation. Situation reports. https:// www.who.int/emergencies/diseases/novel-coronavirus2019/situation-reports/. Accessed 2 April 2020

[2] Armocida B, Formenti B, Ussai S, et al. The Italian health system and the COVID-19 challenge. Lancet Public Health. 2020;2667(20):30074.

[3] Zou L, Ruan F, Huang M, et al. SARS-CoV-2 Viral load in upper respiratory specimens of infected patients. $\mathrm{N}$ Engl J Med. 2020;382(12):1177-9.

[4] van Doremalen N, Bushmaker T, Morris DH, et al. Aerosol and surface stability of SARS-CoV-2 as compared with SARS-CoV-1. N Engl J Med. 2020;382(16): 1564-7.

[5] World Health Organisation. Rational use of personal protective equipment for coronavirus disease (COVID19): interim guidance-2-recommendations for optimizing the availability of PPE. 2020. https://apps.who.int/ iris/bitstream/handle/10665/331215/WHO-2019-nCovIPCPPE_use-2020.1-eng.pdf

[6] Ierardi AM, Wood BJ, Gaudino C, et al. How to handle a COVID-19 patient in the angiographic suite. Cardiovasc Interv Radiol. 2020; in press. doi: 10.1007/s00270020-02476-8 